九州大学学術情報リポジトリ

Kyushu University Institutional Repository

\title{
Identification of full-substorm onset from ground-magnetometer data by singular value transformation
}

Tokunaga, Terumasa

Department of Earth \& Planetary Sciences, Graduate School of Sciences, Kyushu University

Yumoto, Kiyohumi

Space Environment Research Center, Kyushu University

Uozumi, Te i j i

Space Environment Research Center, Kyushu University

CPMN Group

https://doi.org/10.5109/19197

出版情報: 九州大学大学院理学研究院紀要 : Series D, Earth and planetary sciences. 32 (3)， pp.63-73, 2011-03-10. Faculty of Science, Kyushu University

バージョン :

権利関係 : 
Mem. Fac. Sci., Kyushu Univ., Ser. D, Earth \& Planet. Sci., Vol. XXXII, No. 3, pp. 63-73, March 10, 2011

\title{
Identification of full-substorm onset from ground-magnetometer data by singular value transformation
}

\author{
Terumasa Tokunaga*, Kiyohumi Yumoto**, Teiji Uozumi* ${ }^{* *}$ and CPMN Group
}

\begin{abstract}
Pi 2 magnetic pulsations observed on the ground are a good indicator of the auroral breakup. However, they have not only corresponding full-substorm onsets but also most pseudobreakups. Another well-known substorm related phenomenon observed on the ground is positive bays. In order to identify full-substorm onsets from ground-magnetometer data, we developed a new algorithm based on "Singular Spectrum Analysis (SSA)". The algorithm enables us to screen Pi 2 pulsations accompanied by the magnetic positive bay. We applied proposed algorithm to ground-magnetometer data and compared to the obtained results with Polar/UVI data. As a result, we succeeded in identifying $62 \%$ of the full-substorm onsets from ground-magnetometer data obtained in the nighttime sector between 21 and 03LT.
\end{abstract}

Keywords: auroral substorm, Pi 2 pulsation, positive bay, Singular Value Transformation

\section{Introduction}

Pi 2 magnetic pulsations are defined as impulsive hydromagnetic oscillations with a period of 40-150s. It is well-known that Pi 2 pulsations has one-to-one correspondence with auroral breakups (Jacobs et al., 1964; Saito, 1969; Yumoto and CPMN Group, 2001). The excitation of the Pi 2 wave is closely related to the substorm trigger (Saito, 1976) and therefore Pi 2 onsets are often used for identifying substorm onsets. Although Pi 2 pulsations have been accepted as a good indicator of auroral breakups, they correspond to not only full-substorm onsets but also most pseudobreakups (Rostoker et al., 1980). Hsu and McPherron (2007) examined statistical properties of Pi 2 pulsations. They reported that the most probable number of Pi 2 bursts per substorm is 2. Pseudobreakups are characterized by short lifetimes, extreme locations, and weak magnetic perturbations on the ground (McPherron, 1991). In the previous substorm researches, geophysical differences between pseudobreakups and full substorms have been studied (Ohtani et al., 1993; Nakamura et al., 1994; Ohtani et al., 2002; Partamies et al., 2003). However, the physical difference in the magnetosphere and on the ground has not been clarified yet. In other words, to figure out the physical difference is a key subject for understanding the substorm onset mechanism. From this viewpoint, it is important to detect Pi 2 pulsations that correspond to full-substorm onset.

In 1990s, some studies have been made on the automatic detection of ground-observed Pi 2 pulsations. Takahashi et al. (1995) utilized wave power in the Pi 2 frequency band, which is calculated by applying the discrete Fourier transform, for selecting Pi 2 pulsations. An wavelet analysis has also been used to detect Pi 2 pulsations (Nose et al., 1998; Murphy et al., 2009). Recently, Hilbert-Huang Transformation used as ULF (Ultra Low Frequency) wave diagnosis of substorm expansion phase onset (Kataoka et al., 2009). Furthermore, the pattern recognition capabilities of artificial neural networks (ANN) have been used to identify Pi 2 pulsations (Sutcliffe, 2007). However, these methods cannot determine whether the Pi 2 pulsation corresponds to fullsubstorm onsets or to psedobreakups.

Another well-known substorm related phenomenon measured on the ground is positive bays. At the onset of

Manuscript received on 15 December 2010; accepted on 13 January 2011

* Department of Earth \& Planetary Sciences, Graduate School of Sciences, Kyushu University, 6-10-1 Hakozaki, Higashi-ku, Fukuoka 812-8581, JAPAN; Corresponding author's e-mal: tokunaga@geo.kyushu-u.ac.jp

** Space Environment Research Center, Kyushu University, 6-10-1 Hakozaki, Higashi-ku, Fukuoka 812-8581, JAPAN 
the substorm expansion phase, the crosstail current is diverted down the magnetic filed lines. The current then flows in the ionosphere as the westward electrojet and returns to the tail along the magnetic field line. The perturbation of tail current can be represented by an equivalent eastward current, which completes the threedimensional current wedge. The mid-latitude and low-latitude signature of this wedge is a positive perturbation in the north-south component (McPherron et al., 1973a; Clauer and McPherron, 1974). The positive perturbation is called substorm positive bay (Akasofu and Meng, 1969; Meng and Akasofu, 1969). It is widely accepted that the sudden formation of the current wedge is essential to complete substorm onset. Hence, positive bays at mid and low latitudes could be an evidence for the occurrence of full substorms. From these arguments, we can say that Pi 2 pulsations accompanied by positive bays are one of the most reliable indicators of full-substorm onsets.

In order to identify full-substorm onsets from ground-magnetometer data, we propose a new algorithm to screen Pi 2 pulsations that accompanied by positive bays. As mentioned earlier, there are some works about automatic detections of Pi 2 pulsations. However, these methods are limited only to detecting wave packets. To screen Pi 2 pulsations that accompanied by positive bays, we should detect wave packets and changes of slopes simultaneously in ground-magnetometer data. Recently, singular spectrum analysis (SSA) has been used for change-point detections in time series (Moskvina and Zhigljavsky, 2003). Ide and Inoue (2005) developed the SSA-based change-point detection method, named singular spectrum transformations (SST), and showed that it was useful in knowledge discovery of causal relationships from a set of heterogeneous time series. Recently, the SST has been applied to determine the onset of positive bays (Tokunaga et al., 2010a, b). Unlike other conventional approaches, the SSA is data adaptive and does not employ any specific generative models. Further, SSA can extract simultaneously complex trends and periodic components. Hence, SSA-based change-point detection method likely fills our purpose, that is, to detect wave packets and changes of slopes simultaneously. In this paper, we introduce a new SSA-based change-point detection method, named Singular Value Transformation (SVT), to screen Pi 2 pulsations that accompanied by positive bays.

The outline of the rest of the paper is as follows. In Section 2, we describe the basic concept of SSA. Further, we introduce "bay-score" that provides information to determine whether the Pi 2 pulsation accompanied by the positive bay is present or not. Then, we define the framework of SVT. In Section 3, we apply our algorithm to the ground-magnetometer data and compare the result with auroral images obtained by Polar Ultra Violet Imager (Polar/UVI). Furthermore, we evaluated the practical performance of the algorithm in a statistical study.

\section{Singular Value Transformation}

In this section, we introduce the new method, named SVT, to detect substorm positive bays from groundmagnetometer data. The SVT can be regard as an applied technique of SSA. Thus, we first describe the basic concept of SSA.

\section{1. Pattern Extraction by SSA}

First of all, let us consider a transformation of a sequence time series $Y=\left\{y_{1}, y_{2}, \ldots, y_{K}, \ldots, y_{N}\right\}$ into the multidimensional series $\mathbf{X}=\left[X_{1}, X_{2}, \ldots, X_{K}\right]$, where the $X_{i}$ denotes a subsequence that can be described as $X_{i}=\left(y_{i}, \ldots, y_{i+K-1}\right)(1 \leq i \leq L)$. Vectors $X_{i}$ 's and the matrix $\mathbf{X}$ are called L-lagged vectors and an L-trajectory matrix, respectively. Note that an $L$-trajectory matrix $\mathbf{X}$ is an $L \times K$ Hankel matrix described as

$$
\mathbf{X}=\left(\begin{array}{cccc}
y_{1} & y_{2} & \cdots & y_{K} \\
y_{2} & y_{3} & \cdots & y_{K+1} \\
\vdots & \ddots & \vdots & \vdots \\
y_{L} & y_{L+1} & \cdots & y_{N}
\end{array}\right)
$$

We call $K$ and $L$ a window width and an embedding dimension, respectively.

The second step of the SSA is the SVD of the Hankel matrix $\boldsymbol{X}$. Let us denote $\left(\lambda_{1}, \lambda_{2}, \ldots, \lambda_{L}\right)$ as squared singular values of $\mathbf{X X}^{\mathbf{T}}$ in decreasing order of the magnitude $\left(\lambda_{1} \geq \lambda_{2} \geq \cdots \geq \lambda_{L}\right)$. Now the SVD of the Hankel matrix $\boldsymbol{X}$ can be described as $\mathbf{X}=\mathbf{U} \mathbf{L} \mathbf{V}^{\mathrm{T}}$, where $\boldsymbol{\Lambda}$ denotes a diagonal matrix whose diagonal element equal to the squared singular values, $\mathbf{U}$ denotes a left singular matrix and $\mathbf{V}$ denotes a right singular matrix. Superscript $T$ denotes the transpose of a matrix. Then, the Hankel matrix $\mathbf{X}$ can be described as a sum of rank-one bi-orthogonal 
elementary matrices $\mathbf{X}=\mathbf{X}_{1}+\mathbf{X}_{2}+\cdots+\mathbf{X}_{L}$. The $i$ th elementary matrix can be described by using the $i$ th left singular vector and the $i$ th right singular vector as $\mathbf{X}_{i}=\lambda_{i} U_{i} V_{i}^{T}(i=1, \ldots, L)$. A set of these three notations consists of singular value $\lambda_{i}$, empirical orthogonal functions $U_{i}$ and principal components $V_{i}$. Note that $L$ corresponds to the number of singular vectors.

Now let us define representative patterns using empirical orthogonal functions $U_{i}(1 \leq i \leq L)$. As described above, the method to extract dominant structures in time series via the SVD on the Hankel matrix is referred to as the SSA.

\section{2. Preliminary procedure for $S V T$}

Let us consider to create a $L \times K$ Hankel matrix $\hat{\mathbf{X}}$ according to the procedure shown in Figure 1 .

$$
\hat{\mathbf{X}}=\left(\begin{array}{cccc}
y_{1} & y_{2} & \cdots & y_{K} \\
y_{\tau} & y_{\tau+1} & \cdots & y_{\tau+K} \\
\vdots & \ddots & \vdots & \vdots \\
y_{(L-1) \tau+1} & y_{(L-1) \tau+2} & \cdots & y_{(L-1) \tau+K}
\end{array}\right)
$$

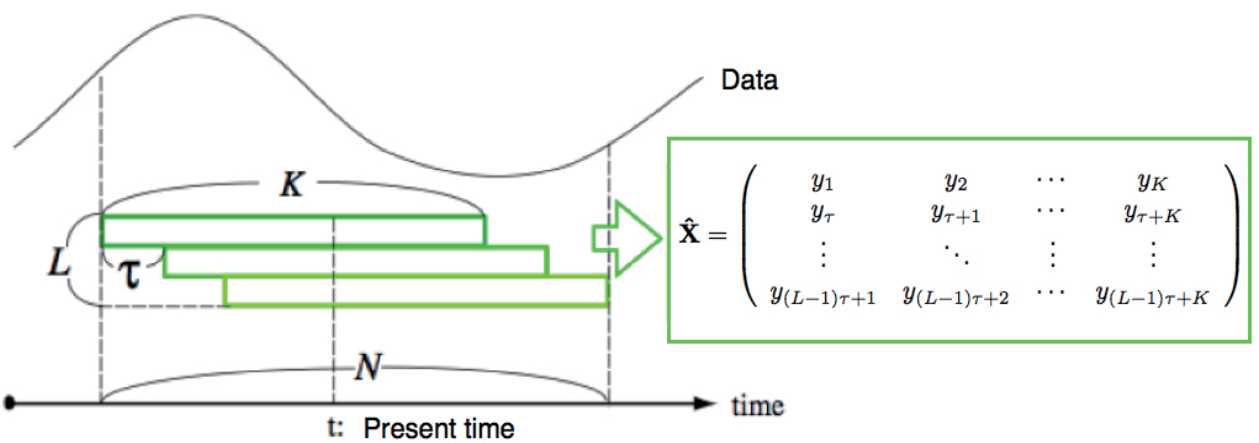

Fig. 1. Schematic illustration of the preliminary procedure for SVT.

Here, we call $K$ and $L$ a window width and an embedding dimension. $\tau$ represents a shift length. Next, we consider a SVD of the matrix $\hat{\mathbf{X}}$. Let us denote $\left(\hat{\lambda_{1}}, \hat{\lambda_{2}}, \ldots, \hat{\lambda_{L}}\right)$ as squared singular values of $\hat{\mathbf{X}} \hat{\mathbf{X}}^{T}$ in decreasing order of the magnitude $\left(\hat{\lambda_{1}} \geq \hat{\lambda_{2}} \geq \cdots \geq \hat{\lambda_{L}}\right)$, where the subscript $T$ denotes transpose of a matrix. Now the SVD of the Hankel matrix $\hat{\mathbf{X}}$ can be described as $\hat{\mathbf{X}}=\hat{\mathbf{U}} \hat{\mathbf{\Lambda}} \hat{\mathbf{V}}^{T}$, where $\hat{\boldsymbol{\Lambda}}$ denotes a diagonal matrix whose diagonal element equal to the squared singular values, $\hat{\mathbf{U}}$ denotes a left singular matrix and $\hat{\mathbf{V}}$ denotes a right singular matrix. For simplicity, now we assume that $\hat{\boldsymbol{\Lambda}}$ is a square matrix, that is, $K=L$. Then, $\hat{\boldsymbol{\Lambda}}$ can be described as

$$
\hat{\boldsymbol{\Lambda}}=\left(\begin{array}{cccc}
\hat{\lambda_{1}} & 0 & \cdots & 0 \\
0 & \hat{\lambda_{2}} & \cdots & 0 \\
\vdots & \ddots & \vdots & \vdots \\
0 & 0 & \cdots & \hat{\lambda_{L}}
\end{array}\right)
$$

Now let the $i$ th column vector of matrix $\hat{\mathbf{U}}$ and the $i$ th row vector of matrix $\hat{\mathbf{V}}$ be $\hat{U}_{i}$ and $\hat{V}_{i}$, respectively. $\hat{U}_{i}$ and $\hat{V}_{i}$ called empirical orthogonal functions and principal components, respectively. The magnitude of $\hat{\lambda}_{i}$ correspond to powers of empirical orthogonal functions and principal components. 
Next, we focus attention on the time variation of $\hat{\lambda}_{i}$. In Figure 2, we show the test data, which we created based on a mid- and low-latitude magnetic variation around a substorm onset. Figure 3a shows the time variation of $\hat{\lambda}_{i}(i=1, \cdots, 20)$. Figure $3 \mathrm{~b}$ shows $\hat{\lambda}_{i}(i=1, \cdots, 20)$ at $t=250 \mathrm{~s}$ and Figure $3 \mathrm{c}$ shows $\hat{\lambda}_{i}(i=1, \cdots, 20)$ at $t=1400 \mathrm{~s}$

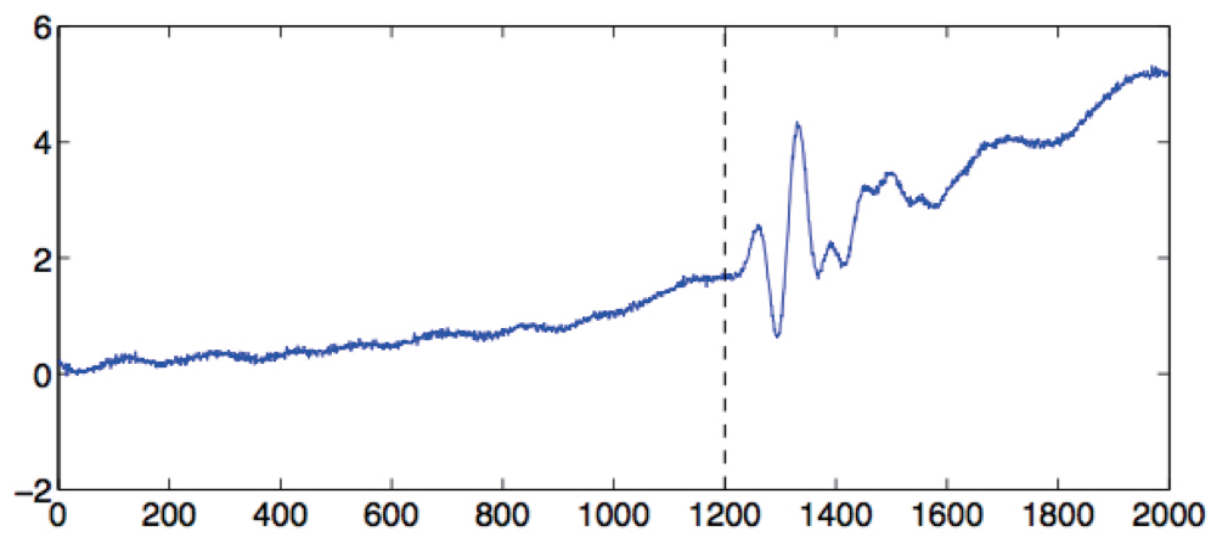

Fig. 2. Test data, which we created based on a mid- and low-latitude magnetic variation around a substorm onset. The vertical dashed line at $\mathrm{t}=1200 \mathrm{~s}$ represents the start time of extraordinary oscillations.

(a)

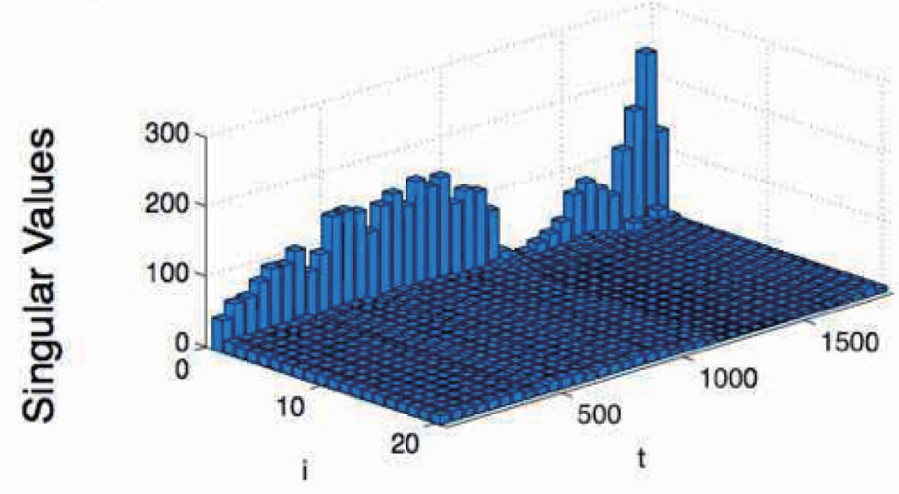

(b)

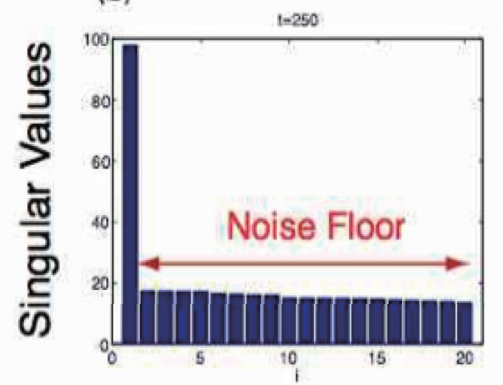

(c)

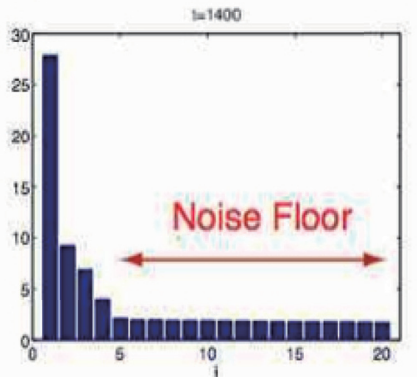

Fig. 3. (a) A time variation of singular values for the test data shown in Fig. 2 calculated with the procedure described in Section 2.2. Hankel matrices were created with $K=80, L=80, M=20, \tau=30$. (b) Singular values at t=250s. (c) Singular values at t=1400s. 
As shown in Figure $3 b$, we see that the first eigenvalue $\hat{\lambda}_{1}$ is extraordinary large. In contrast, $\hat{\lambda}_{i}(i=2, \cdots, 20)$ are almost the same. So it is likely that empirical orthogonal functions $\hat{U}_{i}(i=2, \cdots, 20)$ or principal components $\hat{V}_{i}(i=2, \cdots, 20)$ represent noise. Also, this indicates that the essential structure of the test data around $t=250 \mathrm{~s}$ can be represented by the first empirical orthogonal functions or the first principal component.

Next, let us focus on Figure $3 \mathrm{c}$ which shows $\hat{\lambda}_{i}(i=1, \cdots, 20)$ at $t=1400 \mathrm{~s}$. At this time, the extraordinary oscillation has already begun. We see that the magnitudes of singular values decrease from $i=1$ to $i=4$. However, $\hat{\lambda}_{i}(i=5, \cdots, 20)$ are the almost same. So it is likely that empirical orthogonal functions $\hat{U}_{i}(i=5, \cdots, 20)$ or principal components $\hat{V}_{i}(i=5, \cdots, 20)$ represent noise. This indicates that the essential structure of the test data around $t=1400 \mathrm{~s}$ can be represented by a linear combination of first to fourth empirical orthogonal functions $\hat{U}_{i}$ $(i=1, \cdots, 4)$ or of first to fourth principal components $V_{i}(i=1, \cdots, 4)$. From what we have seen, it is clear that the distribution of singular values obtained by SVD of the Hankel matrix reflects the complexity of the subsequence.

\section{3. Bay-score}

We introduce a relative anomaly metric of time series at the present time, named "bay-score". It provides information to determine whether the Pi 2 pulsation that accompanied by the positive bay is present or not. As we discussed in Section 2.2, the distribution of singular values calculated by SVD of the Hankel matrix reflects the complexity of the subsequence. The top panel of Figure 4 shows H-component of ground-magnetometer data obtained at KAG. The second panel of Figure 4 shows the time variations of the largest singular value calculated by the procedure described in Section 2.2. Similarly, the third panel and the fourth panel of Figure 4 shows the time variations of the second-largest singular value and that of the third-largest singular value, respectively. The vertical dashed lines in red show Pi 2 pulsations accompanied by positive bays selected by visual inspection. We see that singular values around substorm positive bays decreased. By taking account into the feature, we define the bay-score $Z(\mathrm{t})$ as

$$
Z=\frac{1}{\left(\sum_{i=1}^{M} \frac{\hat{\lambda_{i}}}{\left\|\hat{\lambda_{i}}\right\|}\right)^{3}}
$$

Here, $M$ is the number of singular values to be included for the bay-score. The selection of $M$ affects the sensibility of the SVT. $\|\cdot\|$ is the usual Euclidean norm. $\hat{\lambda}_{i}$ is the $i$ th-largest singular value. Note that $Z(t)$ is nondimensional parameter and positive value by definition. The calculation of the bay-score can be viewed also as a nonlinear transformation from an original time-series $\mathcal{T}$ to a new time-series $\mathcal{T}_{v}$, i.e.

$$
\mathcal{T} \rightarrow \mathcal{T}_{v}(K, L, M, \tau)
$$

We call this transformation "Singular Value Transformation (SVT)". The algorithm detects Pi 2 pulsations that accompanied by positive bays, if the bay-score $Z(t)$ be larger than a predetermined threshold value.

\subsection{Choice of parameters}

As shown in Eq. 5, the SVT algorithm includes four parameters, namely, $K, L, M$ and $\tau$. By nature of SVT, these parameters needed to be determined experimentally. In change-point detection methods based on SSA, the choice of $K$ depends on the kind of structural changes to be detected (Moskvina and Zhigljavsky, 2003). A general rule is to choose $K$ reasonably large. However, if we allow small gradual changes in the time series then we could not take $K$ very large. The feature is most likely valid in the SVT. Since the onset of Pi 2 pulsations is gradual, we should not take $K$ as a large value. In this paper, we set to $K=80 \mathrm{~s}$, which is the typical period of Pi 2 pulsations. Remaining parameters, $K=80, L=80, M=20, \tau=30$ were determined empirically. In the following application, these parameters were set to $L=K, M=20, \tau=30$ s. 


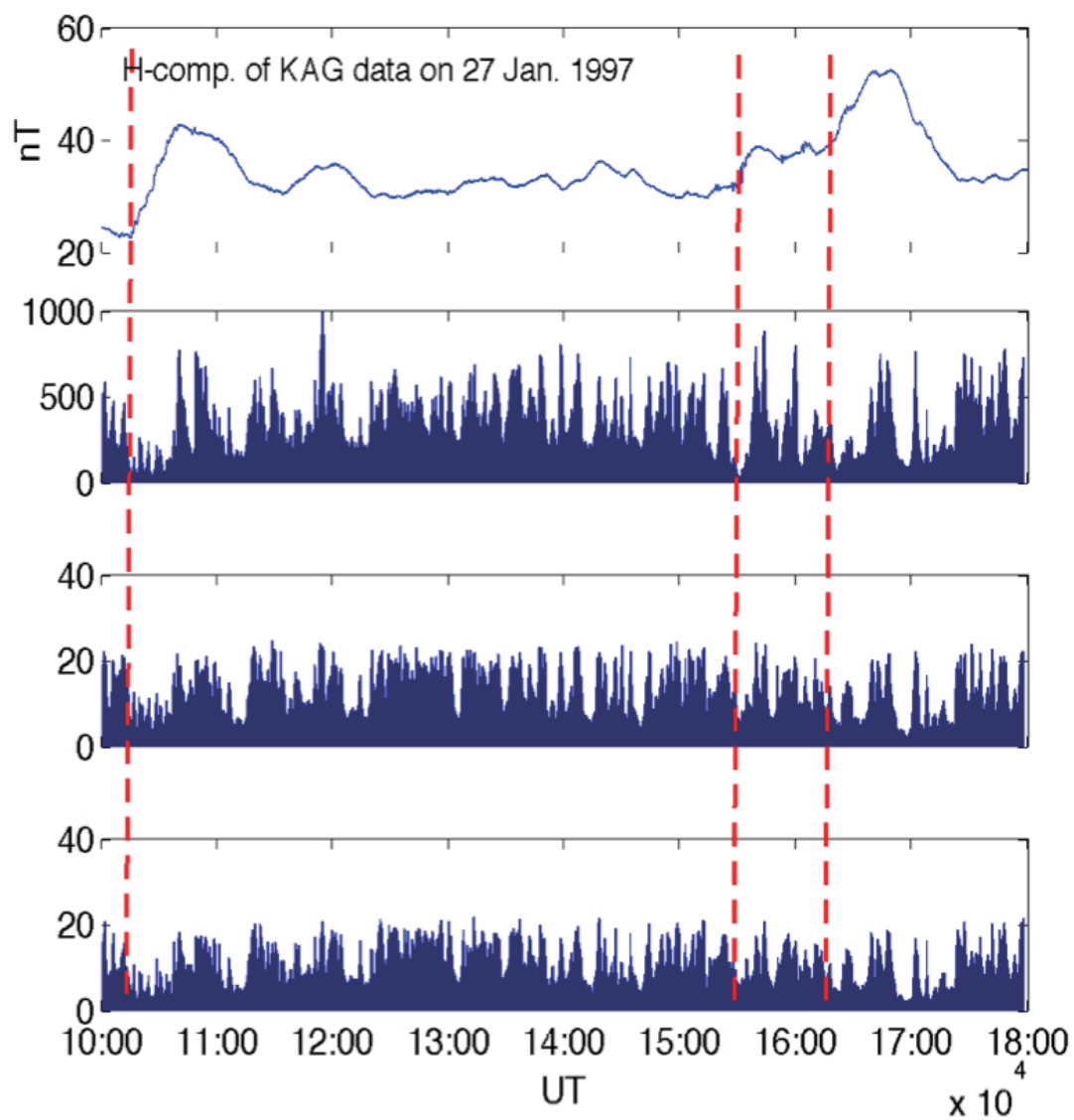

Fig. 4. (Top panel) H-component of ground-magnetometer data obtained at KAG station on 10:00-18:00UT 27 January 1997. The vertical dashed lines in red show the onset of positive bays determined by visual inspection. (Second panel) The largest singular values calculated with $K=80, L=80, M=20, \tau=30$ for the ground-magnetometer data that shown in the top panel. (Third panel) The second-largest singular values. (Fourth panel) The third-largest singular values.

\section{Application to Ground-magnetometer Data}

\section{1. Data Sets}

\section{1. 1. Ground-magnetometer data}

Ground magnetometer data obtained from the CPMN (Circum-pan Pacific Magnetometer Network) stations in the $210^{\circ}$ magnetic meridian (MM) chain (Yumoto and CPMN Group, 2001) have been used in the following applications. CPMN consists of about 50 stations. In this study, the data obtained at KAG were used for the analysis. The locations of these stations are listed in Table 1. The observations were based on vector measurements by fluxgate magnetometers with a sampling rate of $1 \mathrm{~Hz}$.

Table 1. Locations of geomagnetic observatories used in this paper.

\begin{tabular}{|ccc|}
\hline Station & Geographic Latitude & Geographic Longitude \\
\hline KAG & 31.48 & 130.72 \\
\hline
\end{tabular}




\section{1. 2. Polar Ultra Violet Imager}

In this study, Polar Satellite Ultra Violet Imager (Polar/UVI) to check the global auroral activities. The Polar satellite is in a highly elliptical polar orbit with an apogee at about $9 R_{E}$, and it collects data from a high-altitude perspective on auroral activities. In this paper, we referenced the specification of Polar/UVI is described in Liou et al. (1999). UVI is a narrow-angle larger-aperture design. In Figure 5a, we present a sequence of nightside UV auroral images from Polar/UVI. The images were obtained by a combination of Lyman-Birge-Hopfield bands (1400$1600_{A}^{\circ}$ ) on 4 January 1997 . We see that the auroral intensity started to enhance exponentially between 15:45:08UT and 15:46:22UT.

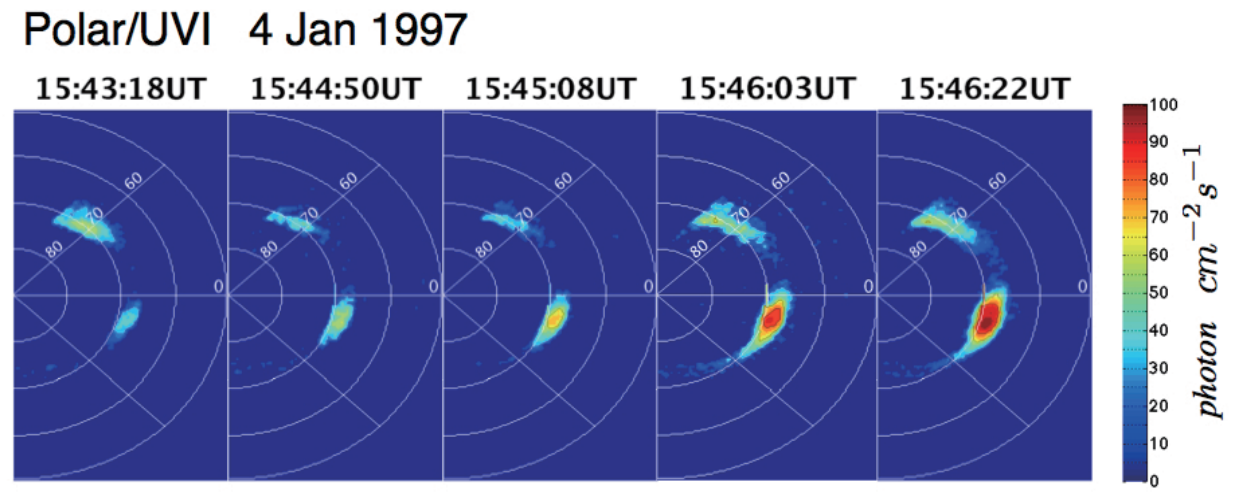

Fig. 5. Polar/UVI images at N2 Lyman-Birge-Hopfield bands (1400-1600 $\stackrel{\circ}{\mathrm{A}})$ on 4 January 1997.

\section{2. Case Studies}

In two case studies, we evaluate the performance of the SVT. The top panel of Figure 6 shows the keogram of auroral brightening observed by Polar/UVI in the Southern Hemisphere on 8 January 1997. The luminescence intensity averaged over magnetic local time of 21-03 h. The vertical axis shows magnetic latitude. The vertical dashed line in red shows full-substorm onsets visually identified by checking the keogram. Here, we define the full-substorm onset as the auroral breakup that is followed by rapid poleward motion of the brightened arc. The middle panel of Figure 6 shows H-component of ground-magnetometer data obtained at KAG station on 8 January 1997. The bottom panel of Figure 6 shows the resulting SVT series calculated with $K=80, L=80, M=20$, $\tau=30$. The dashed horizontal line in blue shows the threshold value $(0.05)$ for the detection of Pi 2 pulsations accompanied by positive bays.

We can see that the result shows two high scores that exceed 0.05 at around 14:20UT and at around 14:40UT. These two high scores most likely correspond to second and third full-substorm onsets shown as vertical dashed lines. However, we see that the first full-substorm onset at around 13:10UT was not detected. This is likely due to the fact that the amplitude of the positive bay at around 13:10UT was small.

Similarly, the top panel of Figure 7 shows the keogram of auroral brightening observed by Polar/UVI in the Southern Hemisphere on 27 January 1997. The middle panel of Figure 7 shows H-component of groundmagnetometer data obtained at KAG station on 27 January 1997. The bottom panel of Figure 7 shows the resulting SVT series calculated with $K=80, L=80, M=20, \tau=30$. The presentation format is the same as Figure 6 . We can see that the result shows two high scores that exceed 0.05 at around 10:00UT, at around 15:30UT, at around 15:15UT and at around 17:10UT. It is likely that four full-substorm onsets were detected successfully by the proposed algorithm. 

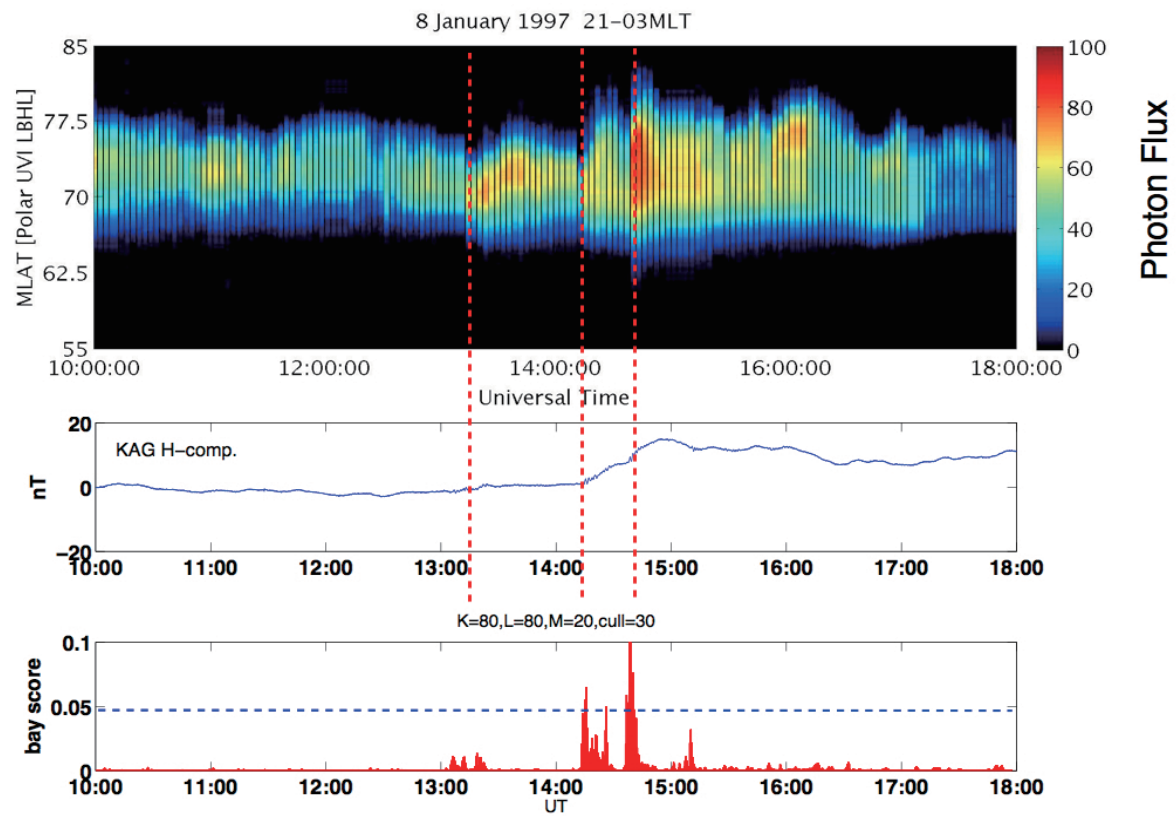

Fig. 6.(Top) Keogram of auroral brightening observed by Polar/UVI in the Southern Hemisphere on 8 January 1997. The luminescence intensity averaged over magnetic local time of 21-03LT. (Middle) H-component of ground-magnetometer data observed at KAG station on 8 January 1997. (Bottom). The resulting SVT series calculated from H-component of KAG data. The SVT parameters were set to $K=80, L=80, M=20, \tau=30$. The dashed horizontal line in blue shows the threshold value for the detection of Pi 2 pulsations accompanied by positive bays.

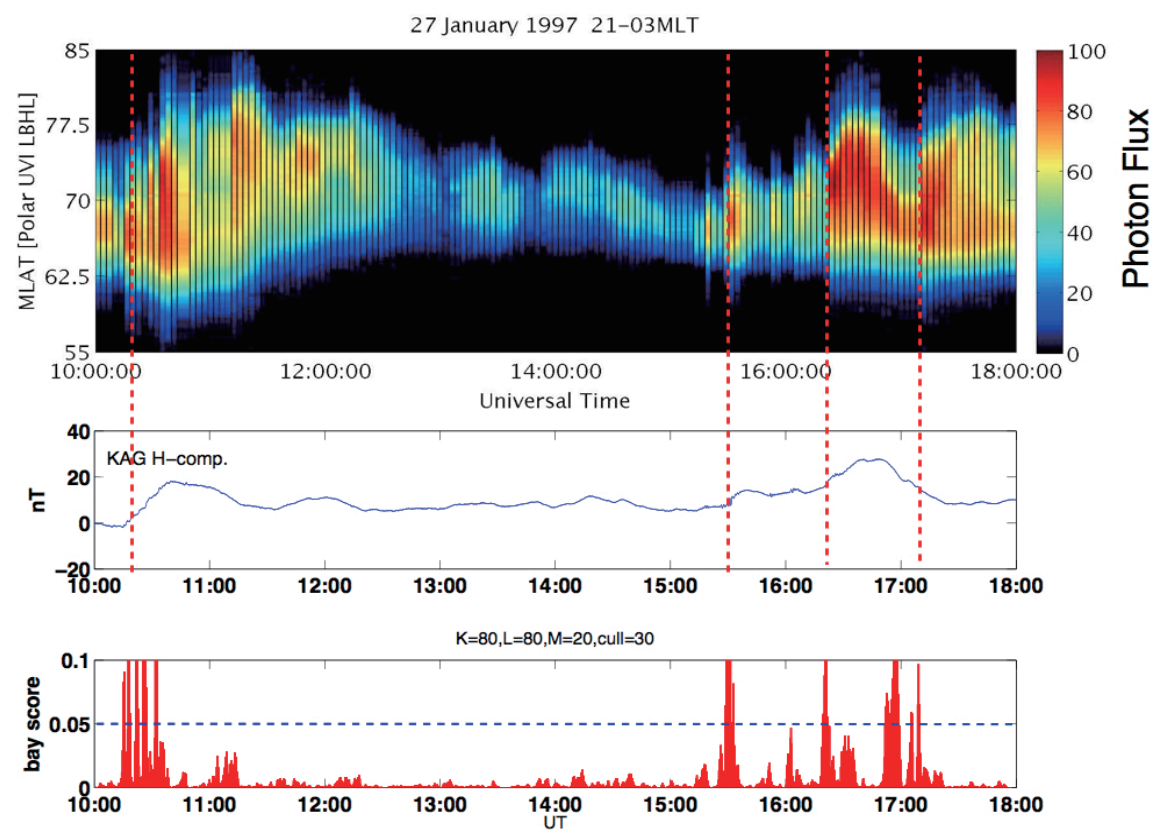

Fig. 7.(Top panel) Keogram of auroral brightening observed by Polar/UVI in the Southern Hemisphere on 27 January 1997. The luminescence intensity averaged over magnetic local time of 21-03LT. (Middle) H-component of ground-magnetometer data observed at KAG station on 27 January 1997. (Bottom panel) The resulting SVT series calculated from H-component of KAG data. The SVT parameters were set to $K=80, L=80, M=20, \tau=30$. The dashed horizontal line in blue shows the threshold value for the detection of Pi 2 pulsations accompanied by positive bays. 


\section{3. Statistical Evaluation}

Next, let us evaluate the performance of the full-substorm onset detection algorithm based on SVT in a statistical study. By checking the keogram of auroral brightening observed by Polar/UVI, we identified 69 auroral breakups for the period from 3 January 1997 through 4 February 1997. We applied the SVT to H-component of ground-magnetometer data obtained at KAG. The investigation was limited in a time period from 10:00-18:00UT, which KAG was located in/around the midnight sector in the period. The SVT parameters were set to $K=80$, $L=80, M=20, \tau=30$. The threshold value for detecting auroral breakups was set to 0.05 . The evaluation procedure is as follows. (1) Detected Events: If the bay-score exceed 0.05 during poleward motion of aurora. (2) False Positive: If the bay-score exceed 0.05 during there is no poleward motion of aurora. (3) False Negative: If the bay-score does not exceed 0.05 during poleward motion of aurora. As we mentioned in Section 1, the most probable number of Pi 2 bursts per substorm is 2 (Hsu and McPherron, 2007). In other words, about half of Pi 2 pulsations is not accompanied by poleward expansion and positive bays. Thus, it is our expectation that the algorithm possibly detect almost equal numbers of full-substorm onsets and false positives.

A test result of the algorithm is summarized in Table 2. The algorithm detected 51 possible full-substorm onsets. Forty three out of 51 events were in good agreement with full-substorm onsets. Eight out of 51 events were false positives. The number of false positive was lower than expected. Twenty six out of 69 events were overlooked. Thus the rate of successful detection is calculated to be about $62 \%$. We consider that our algorithm gives fairly good results on the nightside (21-03LT).

Table 2. Test result of the full-substorm onset detection algorithm based on SVT for the KAG data in the nighttime (21--03LT).

\begin{tabular}{|c|c|c|c|c|}
\hline Date & Event Number & $\begin{array}{l}\text { Successful } \\
\text { Detection }\end{array}$ & False Negative & False Positive \\
\hline 3 Jan. 1997 & 1 & 1 & 0 & 0 \\
\hline 4 Jan. 1997 & 1 & 1 & 0 & 0 \\
\hline 5 Jan. 1997 & 2 & 2 & 0 & 0 \\
\hline 6 Jan. 1997 & 1 & 0 & 1 & 0 \\
\hline 7 Jan. 1997 & 4 & 4 & 0 & 3 \\
\hline 8 Jan. 1997 & 3 & 2 & 1 & 0 \\
\hline 9 Jan. 1997 & 0 & 0 & 0 & 0 \\
\hline 11 Jan. 1997 & 4 & 3 & 1 & 1 \\
\hline 12 Jan. 1997 & 3 & 3 & 0 & 0 \\
\hline 13 Jan. 1997 & 5 & 3 & 2 & 0 \\
\hline 14 Jan. 1997 & 2 & 2 & 0 & 0 \\
\hline 15 Jan. 1997 & 1 & 1 & 0 & 0 \\
\hline 16 Jan. 1997 & 0 & 0 & 0 & 0 \\
\hline 17 Jan. 1997 & 3 & 1 & 2 & 0 \\
\hline 18 Jan. 1997 & 0 & 0 & 0 & 0 \\
\hline 19 Jan. 1997 & 3 & 1 & 2 & 0 \\
\hline 20 Jan. 1997 & 1 & 0 & 1 & 0 \\
\hline 21 Jan. 1997 & 5 & 3 & 2 & 1 \\
\hline 22 Jan. 1997 & 1 & 1 & 0 & 0 \\
\hline 23 Jan. 1997 & 0 & 0 & 0 & 0 \\
\hline 24 Jan. 1997 & 4 & 1 & 3 & 0 \\
\hline 25 Jan. 1997 & 1 & 0 & 1 & 0 \\
\hline 27 Jan. 1997 & 4 & 4 & 0 & 0 \\
\hline 28 Jan. 1997 & 4 & 4 & 0 & 2 \\
\hline 29 Jan. 1997 & 1 & 1 & 0 & 0 \\
\hline 30 Jan. 1997 & 4 & 2 & 2 & 0 \\
\hline 31 Jan. 1997 & 1 & 0 & 1 & 0 \\
\hline 1 Feb. 1997 & 1 & 0 & 1 & 0 \\
\hline 2 Feb. 1997 & 4 & 1 & 3 & 0 \\
\hline 3 Feb. 1997 & 1 & 1 & 0 & 0 \\
\hline 4 Feb. 1997 & 4 & 1 & 3 & 1 \\
\hline Total & 69 & 43 & 26 & 8 \\
\hline
\end{tabular}




\section{Conclusions}

In order to identify the onset of full-substorms from ground-magnetometer data, we developed a new algorithm to detect Pi 2 pulsations that accompanied by positive bays. There are some works about automatic detection of Pi 2 pulsations. However, the previous studies are limited in detecting only wave packets. Thus, we defined the "bay-score" which provides information to determine whether positive bay is present or not. The transformation from an original time-series to a new time series of the bay-score named "Singular Value Transformation (SVT)". We have applied the SVT to ground-magnetometer data and showed that high scores of resulting SVT series are in good agreement with that full-substorm onsets. Further, we evaluated the practical performance of the SVT in a statistical study. By checking the keogram of auroral brightening observed by Polar/ UVI, 69 full-substorm onsets were identified for the period from 3 January 1997 through 4 February 1997. Forty three events of them were detected successfully from ground-magnetometer data by proposed algorithm. On the other hand, 26 events were overlooked. This is mainly due to a local time dependence of positive bays (McPherron, 1973b). As mentioned in Section 1, it is thought that the substorm positive bay is caused by the substorm current wedge. Hence, the positive bay intensity strongly depends the positional relationship between the auroral breakup and the station. If KAG station was located outside the current wedge, the detection of positive bay likely failed regardless of the SVT parameter setting. By using the network data that covers wide range of longitude, the detection ratio will be improved. Whereas, the number of false positive was lower than expected. Hence, we conclude that the proposed algorithm is a strong method to detect positive bays from ground-magnetometer data. In other words, it will enable us to identify full-substorm onsets from groundmagnetometer data.

\section{Acknowledgments}

We would like to thank Prof. J. Dowell of the University of Iowa for providing the Polar/PWI data, Dr. C. Meng and Dr. K. Liou of Johns Hopkins University for providing Polar/UVI data. We thank all the members of the CPMN project (PI; K. YUMOTO) for their ceaseless support. The CPMN project is financially supported by the Ministry of Education, Science, and Culture of Japan (and Japan Society for the Promotion of Science (JSPS)) as the Grants-in-Aid for Overseas Scientific Survey (05041060, 0841105, 10041122, 12373003). The senior author (T. Tokunaga) was supported by the JSPS under Grant-in-Aid for Scientific Research 2005443. We would like to thank Dr. H. Kawano for his great assistance in evaluating and improving this paper as a reviewer.

\section{References}

Akasofu, S.-I., and Meng, C.-I. (1969) A study of polar magnetic substorm. J. Geophys. Res., 74(1), 293-313, doi:10.1029/ JA074i001p00293.

Clauer, C.-R., and McPherron, R.-L. (1974) Mapping the local time-universal time development of magnetospheric substorms using mid-latitude magnetic observations. J. Geophys. Res., 79(19), 2811-2820.

Hsu, T.-S., and McPherron R.-L. (2007) A statistical study of the relation of Pi 2 and plasma flows in the tail. $J$. Geophys. Res., 112 (A5), doi:10.1029/2006JA011782.

Ide, T., and Inoue, K. (2005) Knowledge discovery from heterogeneous dynamic systems using change-point correlations. In Proceedings of 2005 SIAM International Conference on Data Mining, Society for Industrial and Applied Mathematics, 571-576.

Jacobs, J.-A., Kato, Y., Matsushita, S., and Troitskaya, V.-A. (1964) Classification of geomagnetic micropulsations. J.Geophys. Res., 69(1), 180-181.

Kataoka, R., Miyoshi, Y., and Morioka, A. (2009) Hilbert-huang transform of geomagnetic pulsations at auroral expansion onset. J. Geophys. Res., 114, doi: 10.1029/2009JA014214.

Liou, K., Meng, C.-I., Lui, A.-T., and Newell, P.-T. (1999) On relative timing in substorm onset signatures. $J$. Geophys. Res., 104 (A10), 22807-22817.

McPherron, R.-L., Russell, C.-T., and Aubry, M.-P. (1973a) Satellite studies of magnetospheric substorms on August 15, 1968, 9, Phenomenological model for substorms. J. Geophys Res, 78(16), 3131- 3149.

McPherron, R.-L., Russell, C.-T., Kivelson, M.-G., and Coleman Jr., P.-J. (1973b) Substorms in space: The correlation between ground and satellite observations of the magnetic field. Radio Science, 8(11), 1059-1076.

McPherron, R.-L. (1991) Physical process producing magnetospheric substorms and magnetic storms. in Geomagnetism, 4, 593-739.

Meng, C.-I. and Akasofu, S.-I. (1969) A Study of Polar Magnetic Substorms 2. Three-Dimensional Current 
System. J. Geophys. Res., 74(16), 4035-4053, doi:10.1029/JA074i016p04035.

Moskvina, V., and Zhigljavsky, A. (2003) An algorithm based on singular spectrum analysis for change-point detection. Communications in Statistics-Simulation and Computation, 32(2), 319-352.

Murphy, R.-K, Rae, I.-J., Ian, R.-M, Milling, D.-K., Watt, C.-E., Ozeke, L., Frey, H.-U., Angelopoulos, V., and Russel, C.-T. (2009) Wavelet-based ULF wave diagnosis of substorm expansion phase onset. J. Geophys. Res., 114, doi:10.1029/2008JA013548.

Nakamura, R., Baker, D.-N., Yamamoto, T., Belian, R.-D., Bering III, E.-A., Benbrook, J.-R., and Theall, J.-R. (1994) Particle and field signatures during pseudobreakup and major expansion onset. J. Geophys. Res., 99(A1), 207-221.

Nose, M., Iyemori, T., Takeda, M., Kamei, T., Milling, D.-K., Orr, D., Singer, H.-J., Worthington, E.-W., and Sumitomo, N. (1998) Automated detection of Pi 2 pulsations using wavelet analysis: 1. Method and an application for substorm monitoring. Earth, Planetary and Space Sciences, 50(9), 773-783.

Ohtani S., Anderson, B.-J., Sibeck, D.-G., Newell, P.-T., Zanetti, L.-J., Potemura, T.-A., Takahashi, K., Lopez, R.-E., Angelopoulos, V., Nakamura, R., Klumper, D.-M., and Russell, C.-T. (1993) A multisatellite study of a pseudo-substorm onset in the near-Earth magnetotail. J. Geophys. Res., 98(A11), 19355-19367.

Ohtani, S., Yamaguchi, R., Nose, M., Kawano, H., Engebretson, M., and Yumoto, K. (2002) Does the braking of the fast plasma flow trigger a substorm?: a study of the August 14, 1996, event. Geophys. Res. Lett., 29(15), doi: 10.1029/2001GL013785.

Partamies, N., Amm, O., Kauristie, K., Pulkkinen, T.-I., and Tanskanen, E. (2003) A pseudo-breakup observation: Localized current wedge across the postmidnight auroral oval. J. Geophys. Res., 108(A1), doi: 10.1029/ 2002JA009276.

Rostoker, G., Akasofu, S.-I., Foster, J., Greenwald, R.-A., Kamide, Y., Kawasaki, K., Lui, A.-T.-Y., McPherron, R.-L., Russell, C.-T. (1980) Magnetospheric substorms - Definition and signatures. J. Geophys. Res., 85(NA4), 1663-1668.

Sato, T. (1969) Geomagnetic pulsations. Space Science Review, 10 (3), 319-412.

Saito, T., Yumoto, K., and Koyama, K. (1976) Magnetic pulsations pi2 as a sensitive indicator of magnetospheric substorm. Planet. Space Sci., 24(11), 1025-1029, doi:10.1016/0032-0633(76)90120-3.

Sutcliffe, P. R. (2007) Substorm onset identification using neural networks and Pi2 pulsations. Ann. Geophysicae, 15(10), 1257-1264.

Takahashi, K., Ohtani, S., and Anderson, B.-J. (1995) Statistical analysis of Pi 2 pulsations observed by the AMPTE CCE spacecraft in the inner magnetosphere. J. Geophys. Res., 100(A11), 21929-21941.

Tokunaga, T., Ikeda, D., Nakamura, K., Higuchi, T., Yoshikawa, A., Uozumi, T., Fujimoto, A., Morioka, A., and Yumoto, K. (2010a) Detecting precursory events in time series data by an extension of singular spectrum transformation. In: Proceedings of the 10th WSEAS International Conference on Applied Computer Science, Fujita, H., Sasaki, J., and Guizzi, G. (eds.), WSEAS Press, 366-374.

Tokunaga, T., Ikeda, D., Nakamura, K., Higuchi, T., Yoshikawa, A., Uozumi, T., Fujimoto, A., Morioka, A., and Yumoto, K. (2010b) Onset time determination of precursory events in time series data by an extension of singular spectrum transformation. International Journal of Circuits, System and Signal Processing, 5, 46-60.

Yumoto, K., and the CPMN Group. (2001) Characteristics of Pi 2 magnetic pulsations observed at the CPMN stations: a review of the STEP results. Earth Planets Space, 53(10), 981-992. 
